\title{
New light curve analysis and period changes of the overcontact binary $\mathrm{XY}$ Leonis
}

\author{
K. Yakut, C. İbanoğlu, B. Kalomeni, and Ö. L. Değirmenci \\ University of EGE, Faculty of Science, Department of Astronomy and Space Sciences, 35100 Bornova, İzmir, Turkey \\ e-mail: [yakut, ibanoglu, belinda, omerd]@astronomy.sci.ege.edu.tr
}

Received 11 November 2002 / Accepted 5 February 2003

\begin{abstract}
Light curves and photometric solutions of the contact binary XY Leo are presented. The $U B V R$ light curves appear to be almost symmetric. The $B$ and $V$ light curves were analysed with the Wilson-Devinney code and the orbital parameters of the system were obtained and compared with those obtained in previous studies. All the times of minimum light were collected and combined with the new ones obtained in this study. The orbital period of the system oscillates with a period of 19.6 years and a semi-amplitude of 0.023 day. This regular change was analysed under the assumption of the third body hypothesis. The time delay and advance due to the orbit of the eclipsing pair around the third component were computed and subtracted from the original residuals obtained with the linear light elements. The remaining residuals also show a parabolic change. Therefore, we assumed that the orbital period of the system changes with time due to at least two different causes. The parabolic change of the period may be attributed to either mass transfer from less massive component to the more massive one, or an additional component bounded to the double binary system with a longer period.
\end{abstract}

Key words. stars: binaries: eclipsing - stars: individual: XY Leo

\section{Introduction}

The W UMa-type short period contact binary system XY Leonis (BD +18 2307, HIP 49136, $V=9^{m} \cdot 67$, $P=0.28$, K0n) has been subject for many photometric studies in the past several decades since its discovery by Hoffmeister (1934). The first photometric light curve of the binary was obtained in 1956 and published by Koch (1960). Later on, the light curves obtained in 1956 and 1978 were analysed by Koch \& Shanus (1978) using the Wilson \& Devinney (1971) program. They pointed out that the 1978 light curve was considerably more complicated than the earlier ones and the less massive secondary was overluminous for its mass by about $0.5 \mathrm{mag}$ in the mass-luminosity diagram. Having a variable light curve and photometric peculiarities related to the W-type phenomenon XY Leo was considered to be rather active system according to Hilditch (1981) and Kaluzny \& Pojmanski (1983). Season-to-season variability was revealed by Hrivnak (1985) by means of the Fourier analysis of his 1979, 1981 and 1983 light curves. The light-curve asymmetries and variability were attributed to the asymmetric distribution of cool spots or spot regions on one or both components. Since the observed asymmetries in the maxima of XY Leo were less

Send offprint requests to: $\mathrm{K}$. Yakut,

e-mail: yakut@astronomy.sci.ege.edu.tr than 0.04 mag the area covered by spots on the more massive component has been estimated to be less than $8 \%$.

Strong Ca II emission lines were first observed by Struve \& Zebergs (1959). These lines were double at quadratures and narrower with respect to the absorption lines. The reliable radial velocity measurements of this partially eclipsing contact and double-lined spectroscopic binary was obtained by Hrivnak et al. (1984). After corrections for tidal and eclipse effects they derived the masses of the components as 0.87 and 0.44 solar masses. Ca II $\mathrm{H}$ and $\mathrm{K}$ emission was usually strong in the spectrum and had been seen at all orbital phases. They have also noted that this emission seems, in general, to be associated with the more massive component.

The orbital period of the system changes with time. The O-C residuals for XY Leo were studied by Gehlich et al. (1972), Kaluzny \& Pojmanski (1983), Hrivnak (1985) and recently by Pan \& Cao (1998). Gehlich et al. first proposed that the changes of time difference between observed and calculated times of minimum light for this overcontact system may be arisen from a third body, dynamically bounded to XY Leo. Using the mass function they have estimated the minimum mass of the tertiary component as $0.92 M_{\odot}$. Due to the lack of any spectroscopic evidence about the third body, they concluded that it could be a white dwarf. Moreover, they have represented the $\mathrm{O}-\mathrm{C}$ residuals by three different orbital periods under the assumption of sudden changes for which no reason 
was given. Hrivnak (1985) re-analyzed all the available O-C values obtained up to 1984 and suggested that the possibility of abrupt period changes at approximately every ten years might not be ruled out. If there exists a third body in the system it should be a binary composed of equal main-sequence components. Traces of these late type components would not be seen in the spectrum of XY Leo.

Barden (1987) succeded to obtain the spectra of the previously suspected third companion in the binary XY Leo. He revealed the binary nature of this companion composed by two $\mathrm{M}$ dwarf stars with an orbital period of 0.8 day. Moreover, he pointed out that $\mathrm{Ca}$ II $\mathrm{H}$ and $\mathrm{K}$ emission features observed by many investigators were, instead, associated with the components of the BY Draconis type tertiary object. All available times of mid-eclipses were collected by Pan \& Cao (1998). By adding their own times of minimum light they reexamined the $\mathrm{O}-\mathrm{C}$ values. They confirmed the light-time effect and calculated the light-time orbit based on a long database, which covers more than two cycles of the third-body orbit. They have also noted that the additional period change for XY Leo may not be ruled out besides the light-time effect.

The aim of this study is to make more clear the parameters of the third-body orbit, to estimate third-body contribution to the total light and to determine the other plausible causes of the orbital period changes, if there is any.

\section{Observations}

XY Leo was observed photoelectrically with the $48 \mathrm{~cm}$ Cassegrain telescope of Ege University Observatory on six nights during December 2000, January 2001 and May 2002. In 2000 and 2001 the complete light curves of the system were obtained. The light curves obtained in December 2000 have already been published by Yakut \& İbanoğlu (2001). In 2002 the system was observed only for times of the minimum light. The observations were performed with the use of an uncooled SSP-5 type photometer and standard $U, B, V$ and $R$ filters. The comparison star chosen was $\mathrm{BD}+18^{\circ} 2306$, which was used in most previous studies of the system either as a comparison or check star. The differential observations, in the sense variable minus comparison, were corrected for the atmospheric extinction using the extinction coefficients obtained for each night from the brightness variation of the comparison star. The mean errors of differential magnitudes do not exceed 0.01 for four filters,being the scattering in the $U$ passband the largest. Therefore $U$ band light curve was not considered for the light curve analysis. Hrivnak (1985) analyzed the light curves obtained in two passbands, which are close to $B$ and $V$ we used. To compare the results of the light curve solutions we analyzed only the $B$ and $V$ light curves. The complete light curves of the system, obtained at four passbands in 2000 and 2001, are shown in Fig. 1 as $\Delta m$ versus phase. The two minima of the light curve are almost symmetrical in all passbands.

The phases were calculated using the following ephemeris given by Pan \& Cao (1998),

HJD MinI $=2435484.0283+0.28410260 \times E$.

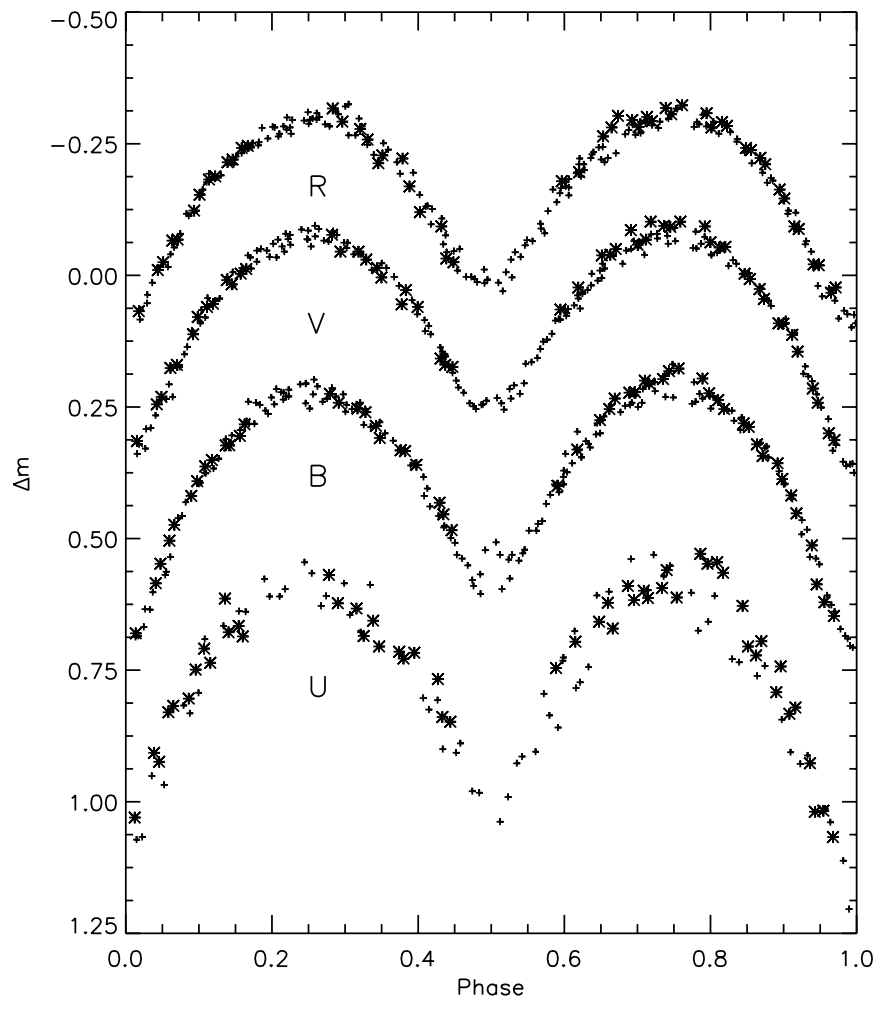

Fig. 1. The light curves of XY Leonis. The plusses indicate the observations obtained by Yakut \& İbanoğlu (2001) in December 2000 and asterisks show the observations obtained in 2001.

Seven heliocentric times of minimum light were obtained using the method of Kwee \& van Woerden (1956) and are given in Table 1 together with the previously obtained timings.

\section{Light curve variations and orbital analysis}

In order to model the $B$ and $V$ band light curves of the system we used the Wilson-Devinney code (Wilson \& Devinney 1971; Wilson 1994) which includes a new reflection treatment, the option of using non-linear limb-darkening laws and the ability to adjust spot parameters. In this method for light curve analysis, some parameters of the components should be fixed. These parameters may be estimated from the known characteristics of the stars. The adopted parameters in the solutions are described as follows: a temperature of $4850 \mathrm{~K}$ for star1 (the star eclipsed at Min I) which corresponds to the $B-V$ colour of 0.82 (Popper 1980), the limb darkening coefficients $x_{1}=x_{2}=0.873$ for $B$ and 0.786 for $V$ band (Al-Naimiy 1978) the values of the gravity darkening coefficients $g_{1}=g_{2}=0.32$ (Lucy 1967) and the values of the albedo $A_{1}=A_{2}=0.5$ (Rucinski 1969). Since the results of previous light curve analysis are in hand we used Mode 3, which assumes both of the components fill their Roche lobes. As we discussed in previous section XY Leo has a third companion. Such a body will affect the depth and slopes of the minima. Therefore, the light curves were analyzed by assuming that there was a third-light in the system. The adjustable parameters are the orbital inclination, $i$; the mean temperature of Star 2, $T_{2}$; the potential of the components, $\Omega_{1}$ and $\Omega_{2}$; the monochromatic luminosity of Star $1, L_{1}$ (the Planck function is 
Table 1. Times of the primary (I) and secondary (II) minima in $\mathrm{JD}_{\text {hel }}^{*}$ $\left(\mathrm{JD}_{\text {hel }}-2400000\right)$.

\begin{tabular}{|c|c|c|c|c|c|c|c|c|c|c|c|}
\hline $\mathrm{JD}_{\text {hel }}^{*}$ & Min. & Ref. & $\mathrm{JD}_{\text {hel }}^{*}$ & Min. & Ref. & $\mathrm{JD}_{\text {hel }}^{*}$ & Min. & Ref. & $\mathrm{JD}_{\text {hel }}^{*}$ & Min. & Ref \\
\hline $31162.258^{*}$ & I & 1 & 44277.7310 & II & 14 & 47969.3226 & II & 23 & 49783.2102 & I & 25 \\
\hline 33005.6250 & II & 2 & 44640.8137 & II & 14 & 47969.4633 & I & 23 & 49783.2127 & I & 25 \\
\hline $33009.617^{*}$ & II & 3 & 44640.9551 & & 14 & 47969.4653 & I & 23 & 49784.0652 & & 25 \\
\hline 33011.5931 & II & 2 & 44665.8135 & II & 14 & 47969.4664 & I & 23 & 49784.2035 & II & 25 \\
\hline $34422.598^{*}$ & I & 4 & 44694.3710 & & 15 & 48224.4470 & II & 24 & 49785.1977 & I & 25 \\
\hline 35195.6628 & I & 2 & 44702.4638 & II & 15 & 48224.4477 & II & 24 & 49828.3876 & I & 27 \\
\hline 35484.0337 & I & 5 & 44706.4411 & II & 15 & 48225.4393 & I & 24 & 50100.4199 & II & 28 \\
\hline 35513.8652 & I & 5 & 45017.5286 & II & 15 & 48225.4399 & I & 24 & 50114.4851 & I & 28 \\
\hline 35517.8430 & I & 5 & 45056.4514 & II & 15 & 48226.4348 & II & 24 & 50114.6255 & II & 28 \\
\hline 35517.9848 & II & 5 & 45074.3486 & II & 15 & 48226.4351 & II & 24 & 50137.6365 & II & 26 \\
\hline 35534.8892 & I & 5 & 45079.7459 & II & 14 & 48723.1966 & I & 25 & 50137.6372 & II & 26 \\
\hline 35564.4364 & I & 6 & 45080.7408 & I & 14 & 48723.1969 & I & 25 & 50152.4114 & II & 28 \\
\hline 35571.3978 & II & 6 & 45396.6566 & I & 16 & 48723.1999 & I & 25 & 50152.5556 & I & 28 \\
\hline 35574.6646 & I & 5 & 45416.6904 & II & 16 & 49088.2805 & I & 25 & 50173.4305 & II & 29 \\
\hline 35576.3717 & I & 6 & 45442.8238 & II & 14 & 49089.2734 & II & 25 & 50180.3974 & I & 29 \\
\hline 35594.6941 & II & 5 & 45444.6685 & I & 16 & 49447.1090 & I & 25 & 50195.3114 & II & 27 \\
\hline 36296.5812 & I & 5 & 45449.6432 & II & 16 & 49447.1099 & I & 25 & 50196.3080 & I & 27 \\
\hline 36297.5756 & II & 5 & 45453.7599 & I & 14 & 49447.2538 & II & 25 & 50482.4031 & I & 28 \\
\hline $39553.492^{*}$ & I & 7 & 45732.8868 & II & 17 & 49447.2539 & II & 25 & 50482.5468 & II & 28 \\
\hline $39609.466^{*}$ & I & 7 & 45740.8414 & II & 14 & 49448.1026 & II & 25 & 50508.3982 & II & 27 \\
\hline $39834.608^{*}$ & II & 7 & 45810.4500 & II & 18 & 49448.1061 & II & 25 & 50513.3698 & I & 27 \\
\hline 41005.5351 & I & 8 & 46079.9122 & & 17 & 49448.2469 & I & 25 & 50519.3375 & I & 30 \\
\hline 41015.3355 & II & 8 & 46469.4080 & & 19 & 49448.2486 & I & 25 & 50519.3377 & I & 27 \\
\hline 41022.2981 & I & 8 & 47275.4007 & & 20 & 49452.0853 & II & 25 & 50519.4 & II & 30 \\
\hline 41022.4387 & II & 8 & 47609.3629 & II & 21 & 49452.2246 & I & 25 & 50519. & & 30 \\
\hline 41062.3571 & I & 8 & 47613.3416 & II & 21 & 49452.2248 & I & 25 & 50539.3671 & II & 30 \\
\hline 41067.4716 & I & 8 & 47626.4092 & II & 21 & 49454.2123 & & 25 & 50547.3 & II & 27 \\
\hline 42051.61956 & I & 9 & 47648.4 & & 21 & 4945 & & 25 & 50862 & II & 27 \\
\hline 42099.48964 & II & 9 & 47864.9146 & & 22 & 49455.0 & & 25 & 50862 & & 27 \\
\hline 42841.4 & I & 10 & 47868.6 & & 23 & 49455 . & & 25 & 50916 & II & 31 \\
\hline 43193.7325 & I & 11 & 47881.8187 & II & 22 & 49456.0604 & II & 25 & 51256.4456 & II & 32 \\
\hline 43193.8760 & II & 11 & 47891.6214 & I & 23 & 49456.0622 & II & 25 & 51257.4389 & I & 33 \\
\hline 43198.7050 & II & 11 & 47896.5933 & II & 23 & 49456.2000 & I & 25 & 51267.3 & I & 32 \\
\hline 43198.8465 & I & 11 & 47897.5881 & I & 23 & 49456.2054 & I & 25 & 51550. & II & 33 \\
\hline 43567.7564 & II & 12 & 47898.5811 & II & 23 & 49756.3626 & II & 26 & 51550. & I & 33 \\
\hline 43572.7288 & I & 12 & 47899.4337 & II & 23 & 49756.3649 & II & 26 & 51884.5888 & II & 34 \\
\hline 43606.3960 & II & 13 & 47899.5763 & I & 23 & 49778.5232 & II & 26 & 51885.5831 & & 34 \\
\hline 43612.6462 & II & 12 & 47928.4124 & II & 21 & 49778.5237 & II & 26 & 51903.4814 & & 34 \\
\hline 43948.7408 & II & 14 & 47928.5545 & & 23 & 49779.2350 & & 25 & 51903.6236 & II & 34 \\
\hline 43949.7367 & I & 14 & 47928.5551 & & 23 & 49782.0704 & & 25 & 51930.4709 & & 34 \\
\hline 43952.7180 & II & 14 & 47928.6963 & II & 23 & 49782.0716 & & 25 & 52337.4 & II & 34 \\
\hline 43953.7144 & I & 14 & 47948.4425 & & 23 & 49782.2148 & II & 25 & 52407.3355 & II & 34 \\
\hline 43959.6794 & I & 14 & 47968.3300 & & 23 & 49782.2157 & II & 25 & & & \\
\hline 43960.6728 & II & 14 & 47968.4712 & II & 23 & 49783.0664 & II & 25 & & & \\
\hline 44239.8053 & & 14 & 47969.3215 & & 23 & 49783.0708 & & 25 & & & \\
\hline
\end{tabular}

References: 1 - Zessewitch (1948); 2 - Koch (1956); 3 - Ashbrook (1952); 4 - Ashbrook (1953); 5 - Koch (1960); 6 - Hinderer (1960); 7 - Locher (1973); 8 - Gehlich et al. (1972); 9 - Burchi \& Zavatti (1975); 10 - Pohl \& Kizllırmak (1977); 11 - Hilditch (1981); 12 - Koch \& Shanus (1978); 13 - Pohl \& Gülmen (1981); 14 - Hrivnak (1985); 15 - Kaluzny \& Pojmanski (1982); 16 - Faulkner \& Grossoehme (1983); 17 - Faulkner (1986); 18 - Pohl et al. (1985); 19 - Pohl et al. (1987); 20 - Keskin \& Pohl (1989); 21 - Wunder et al. (1992); 22 - Mullis \& Faulkner (1991); 23 - Krzesinski et al. (1990); 24 - Markova (1994); 25 - Pan \& Cao (1998); 26 - Agerer \& Heubscher (1996); 27 - Kiss et al. (1999); 28 - Ogloza (1997); 29 - Agerer \& Heubscher (1997); 30 - Agerer \& Heubscher (1998); 31 - Agerer \& Heubscher (1999); 32 - Agerer \& Heubscher (2000); 33 - Ogloza et al. (2000); 34 - This study.

* Visual data.

used to compute the luminosity) and third light, $l_{3}$. Initial value of $l_{3}$ was taken as $8 \%$ for both light curves. $B$ and $V$ light curves were analysed simultaneously. The results obtained after several dozen iterations with DC code are given in Table 2 with those assumed parameters. The filling factor, $f=0.067$, has here the expression $\left(\Omega_{\text {in }}-\Omega\right) /\left(\Omega_{\text {in }}-\Omega_{\text {out }}\right)$ and varies from 0
Table 2. The parameters found by the $B$ and $V$ light curves analysis for XY Leonis.

\begin{tabular}{llllll}
\hline \hline Element & Value $\sigma$ & Element & Value & $\sigma$ \\
\hline$i\left(^{\circ}\right)$ & 68 & \pm 1 & $x_{1_{B}}=x_{2_{B}}$ & $0.87^{*}$ & - \\
$T_{1}(\mathrm{~K})$ & $4850^{*}-$ & $x_{1_{V}}=x_{2_{V}}$ & $0.79^{*}$ & - \\
$T_{2}(\mathrm{~K})$ & 4524 & \pm 14 & $r_{1}$ (pole) & $0.3182 \pm 0.0006$ \\
$\Omega_{1}=\Omega_{2}$ & $4.71 \pm 0.01$ & $r_{1}$ (side) & $0.3331 \pm 0.0007$ \\
$q$ & $1.64^{*}-$ & $r_{1}$ (back) & $0.3671 \pm 0.0010$ \\
$\left(\frac{L_{1}}{L_{1}+L_{2}}\right)_{B}$ & $0.507 \pm 0.043$ & $r_{2}$ (pole) & $0.4012 \pm 0.0005$ \\
$\left(\frac{L_{1}}{L_{1}+L_{2}}\right)_{V}$ & $0.483 \pm 0.043$ & $r_{2}$ (side) & $0.4252 \pm 0.0097$ \\
$\left(l_{3}\right)_{B}$ & $0.019 \pm 0.007$ & $r_{2}$ (back) & $0.4554 \pm 0.0009$ \\
$\left(l_{3}\right)_{V}$ & $0.059 \pm 0.007$ & & & \\
\hline
\end{tabular}

* Assumed.

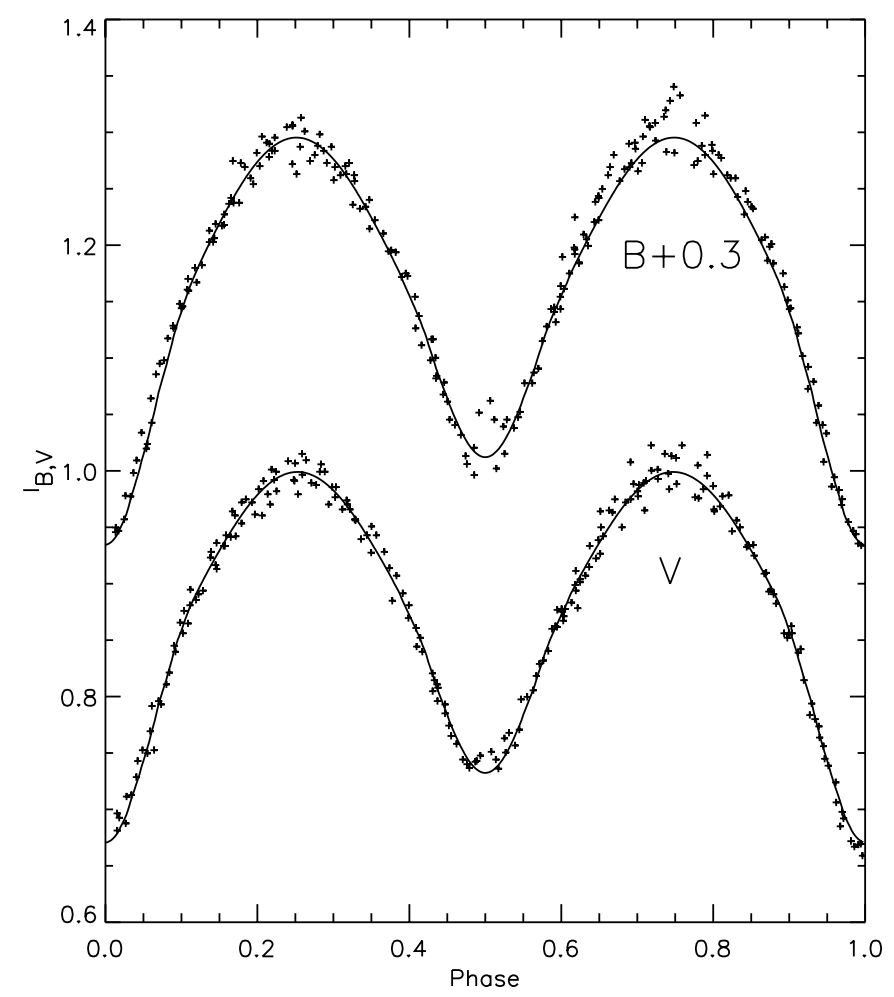

Fig. 2. The observed and computed light curves for XY Leonis.

to unity from the inner to the outer critical surface. This solution indicates that XY Leo is a W-subtype W UMa binary with shallow contact degree. The light curve computed using the parameters given in Table 2 is shown by the solid lines in Fig. 2 and compared with all the observations. The main difference between our solutions and Hrivnak's (1985) is the orbital inclination of the eclipsing pair. Since we used third light as an adjustable parameter the inclination found by our analysis should be larger than Hrivnak's value. We found the orbital inclination of $67^{\circ} .6$, while Hrivnak gives a value of between $65^{\circ} .3$ and 66.5 . The analysis of the light curves indicates that light contribution of the third body is about $2 \%$ in $B$ and $6 \%$ in the $V$-light curve. 


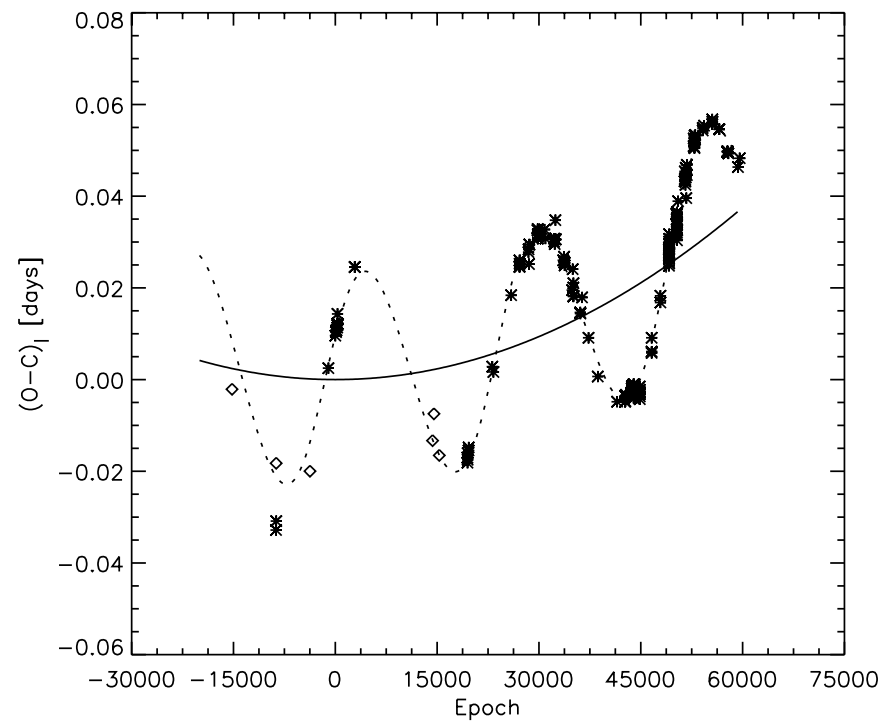

Fig. 3. The $(\mathrm{O}-\mathrm{C})_{\mathrm{I}}$ diagram of the times of mid-eclipses for XY Leo, constructed using the light elements given in Eq. (1). The dashed line represents the $\mathrm{O}-\mathrm{C}$ variation due to the third-body orbit, while the continuous line indicates a parabolic variation superimposed on the variation due to the third-body.

\section{Eclipse timings and period study}

All the timings of the eclipses obtained up to date were collected from the publications of many observers and listed in Table 1 together with newly obtained by us. The $(\mathrm{O}-\mathrm{C})_{\mathrm{I}}$ residuals, indicating the differences between the observed times of eclipses and calculated ones using the light elements given in Eq. (1), were performed. These residuals for all the times of minimum light of XY Leo are plotted versus epoch numbers in Fig. 3. A first glance at the Fig. 3, a sine-like change superimposed on a parabolic variation is clearly seen. The change of the $\mathrm{O}-\mathrm{C}$ residuals reveals that it is a result of two different causes. Therefore, we can compute the times of minimum light with a formula such as

$$
\begin{aligned}
\operatorname{Min} I= & T_{\mathrm{o}}+P E+Q E^{2}+\frac{a_{12} \sin i}{c} \\
& \times\left[\frac{1-e^{2}}{1+e \cos v} \sin (v+\omega)+e \sin \omega\right] .
\end{aligned}
$$

While the last term represents the effect of a third-body, the third term indicates a parabolic variation. The weights were attributed 10 for photoelectric timings and 1 for visual ones. By applying the weighted least-squares solution we obtained the parameters for the third body orbit listed in Table 3 with their standard deviations. The $(\mathrm{O}-\mathrm{C})_{\mathrm{II}}$ residuals were obtained, after subtraction of parabolic and sinusoidal changes, and are plotted versus epoch numbers in Fig. 4.

Using the $a_{12} \sin i$ of $4.083 \mathrm{AU}$ and a period of $19.59 \mathrm{yr}$, the mass function was calculated as $0.177 M_{\odot}$. One can obtain the mass of each component of the eclipsing pair using the semiamplitude of its velocity curve. The semiamplitudes were derived by Barden (1987) as 124.1 and $204.7 \mathrm{~km} \mathrm{~s}^{-1}$ for the more and less massive components, respectively. Therefore we derive the masses of $0.82 M_{\odot}$ and $0.50 M_{\odot}$. Since the total

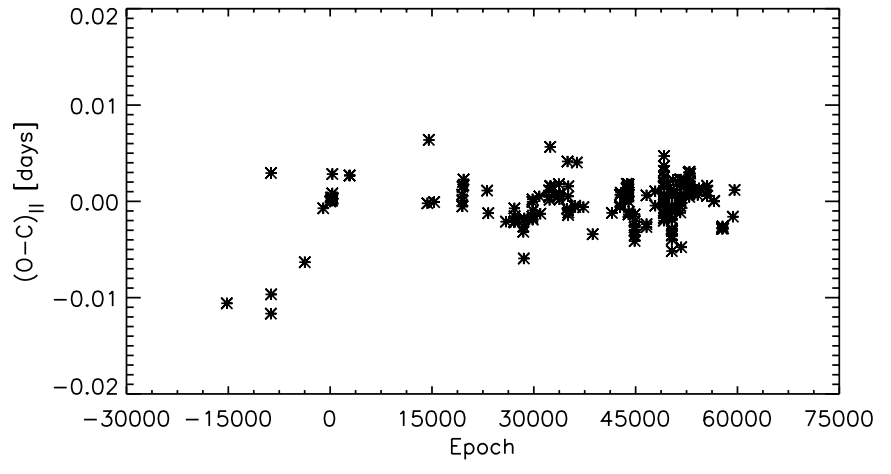

Fig. 4. The $(\mathrm{O}-\mathrm{C})_{\text {II }}$ residuals after subtraction of parabolic and sinusoidal changes.

mass of eclipsing pair was known as $1.32 M_{\odot}$, and the eclipsing pair orbits around the common center of mass with a period of $19.59 \mathrm{yr}$, the mass of the third star may be evaluated from the mass function. It depends, of course, on the inclination of the long period orbit. Specifically we find $0.98(\mp 0.01) M_{\odot}$ for an inclination of $90^{\circ}$. Barden (1987) revealed binary nature of this third-body and obtained the radial velocities of the third and fourth components succesfully. He also derived $m_{3} \sin ^{3} \mathrm{i}$ and $m_{4} \sin ^{3} i$ as 0.066 and $0.047 M_{\odot}$, respectively. If the light-time orbit has an inclination of $90^{\circ}$ then the inclination of this binary should be about $29^{\circ}$. Assuming an inclination of $29^{\circ}$ we estimate the masses of the third and fourth components as 0.57 and $0.41 M_{\odot}$, respectively. The bolometric luminosities of the third-body's components have been estimated from the mass-luminosity diagram of low-mass stars given by Dorman et al. (1989). Their Fig. 2 gives $\log L / L_{\odot}$ of -1.17 and -1.67 for the tertiary and quaternary components of the companion binary, respectively. Then, the luminosities of the third and fourth components are approximately $0.07 L_{\odot}$ and $0.02 L_{\odot}$. Since the luminosities of the eclipsing pair's components were derived by Hrivnak (1985), by using the Popper's (1980) observational data for low-mass stars, as $L_{1}=0.31 L_{\odot}$ and $L_{2}=0.21 L_{\odot}$ the total luminosity of the XY Leonis system should be about $0.61 L_{\odot}$, which is in agreement with that of given by Barden (1987). Applying the bolometric corrections (Popper 1980) we find the percentage contribution of light from each component at $V$ passband to be $54 \%, 40 \%, 5 \%$ and $1 \%$.

On the other hand, the analysis of $\mathrm{O}-\mathrm{C}$ variation yields the coefficient of the quadratic term in Eq. (2) as $(1.04 \mp 0.01) \times$ $10^{-11}$ day per cycle. The orbital period of the eclipsing pair increases with an amount of $2.7 \times 10^{-8}$ day/year. Such an increment in the orbital period may be either due to mass transfer from less massive to the more massive component or due to an additional component to the double binary system with a longer period.

\section{Discussion}

$\mathrm{XY}$ Leo is a W-subtype W UMa contact binary, i.e. the primary eclipse is the occultation but the secondary eclipse is the transit. The eclipsing pair has a mass ratio of 0.6 in a state of marginal contact and a temperature difference of about $326 \mathrm{~K}$. 
Table 3. Orbital elements of the quadrupole system. The standard errors $\sigma$ in the last digit are given in parentheses.

\begin{tabular}{llll}
\hline \hline Parameter & Gehlich et al. (1972) & Pan \& Cao (1998) & This study \\
\hline$T_{1}(\mathrm{HJD})$ & $2435484.0222(16)$ & $2435484.0209(7)$ & $2435484.0239(2)$ \\
$P_{1}($ day) & $0.28410282(7)$ & $0.28410277(3)$ & $0.28410229(1)$ \\
$P_{3}$ (day) & $7274(179)$ & $7112(29)$ & $7156(49)$ \\
$T_{3}(\mathrm{HJD})$ & $2434692(413)$ & $2434437(266)$ & $2434988(25)$ \\
$e$ & $0.13(0.12)$ & $0.17(3)$ & $0.12(1)$ \\
$\omega\left({ }^{\circ}\right)$ & $342(20)$ & $331(13)$ & $356(1)$ \\
$a_{12} \sin i(\mathrm{~km})$ & $6.96(18) \times 10^{8}$ & $5.86(9) \times 10^{8}$ & $6.108(8) \times 10^{8}$ \\
$f(m)\left(M_{\odot}\right)$ & 0.257 & $0.161(1)$ & $0.177(2)$ \\
\hline
\end{tabular}

Table 4. Absolute parameters of XY Leo.

\begin{tabular}{lll}
\hline \hline Parameter & Large star & Small star \\
\hline Mass $\left(M_{\odot}\right)$ & $0.82(2)$ & $0.50(1)$ \\
Radius $\left(R_{\odot}\right)$ & $0.85(2)$ & $0.68(2)$ \\
Effective temperature $(\mathrm{K})$ & $4524(14)$ & 4850 \\
Luminosity $\left(L_{\odot}\right)$ & 0.267 & 0.226 \\
$M_{\text {bol }}$ & 6.2 & 6.4 \\
$M_{v}$ & 6.7 & 6.7 \\
Distance $(\mathrm{pc})$ & \multicolumn{2}{c}{$63_{-6}^{+8}$} \\
\hline
\end{tabular}

The fill-out factor of $7 \%$ was somewhat low for W UMa Wtype binaries, which usually fall in the $10 \%$ to $20 \%$ range. The low fill-out factor indicates that XY Leo may have recently come into contact. The large temperature difference between the components confirms this result. Most theories of the evolution of contact binaries predict that mass ratio decreases with increasing contact age. The spectroscopic mass ratio of the system is about 0.61 (Barden 1987). This value of the mass ratio is in support of the notion that it has recently come into contact. Using the semiamplitudes of the radial velocities of the eclipsing pair's components given by Barden (1987) and the orbital period of the system one can easily obtain $a_{1} \sin i$, $a_{2} \sin i, m_{1} \sin ^{3} i$ and $m_{2} \sin ^{3} i$. Combining these values with those found from light curve analysis the absolute dimensions of the components can be obtained. In Table 4 the absolute parameters of XY Leo are given. For solar values we have taken $T_{\text {eff }}=5780 \mathrm{~K}, M_{\text {bol }}=4.75$ and $B C=-0.14$. The bolometric corrections for the components of eclipsing pair were selected from the tabulation of Popper (1980) as -0.53 and -0.37 for more massive and less massive components, respectively. We find that the mass for massive component is reduced, while the mass for less massive one is increased according the new radial velocity curves obtained by Barden (1987). On the other hand, luminosities of the components are different from those obtained by Hrivnak (1985). The luminosity of the more massive component is $14 \%$ smaller than that found by Hrivnak. Using Table 4 and luminosities of third and fourth components we estimate light contribution of each component to the total light as $47 \%, 47 \%, 5 \%$ and $1 \%$. While the light contribution of the more massive component, is decreasing, the less massive component contributes greater light in $V$ band. However, the light contribution of the third-star does not change, it contributes about $6 \%$, which is in a good agreement with that found from the light curve analysis.
The less massive, smaller component of XY Leo seems to be overluminous and of larger radius with respect to its mass in the mass-luminosity and mass-radius diagrams given by Dorman et al. (1989). While the bolometric absolute magnitude of the more massive component is in agreement with that of low mass stars presented by Popper (1980) and Dorman et al. (1989), the low mass component is brighter about $1.6 \mathrm{mag}$. This discrepancy was discussed by Hrivnak (1985) and concluded that this result is due to the energy transfer from more massive component to the less massive one. Recently, Wang (1994) attempted to explain the W-type phenomenon, i.e., why the smaller component is the hotter one, by using thermal-relaxation-oscillation theory developed by Lucy (1976) and Lucy \& Wilson (1979). The A-type W UMa system is explained as the oscillation phase, the secondary component is expanding toward its equilibrium configuration slowly, while the W-type phenomenon corresponds to the phase in which the secondary component is shrinking toward its ZAMS radius. Thus, the overluminous secondary component is explained by the conversion of the gravitational energy into heat during its contraction. Actually, W-types are always found in a state of near or shallow contact, while A-types have larger fill-out factors.

\section{Conclusions}

New $B$ and $V$ light curves of the contact binary XY Leo were analysed by Wilson-Devinney code. The light contribution of the third body was recalculated for $B$ and $V$ passbands. The orbital inclination, temperature of the cooler component and fractional radii of the components were derived. The results of this analysis were compared with those obtained previously by Hrivnak (1985). Combining the parameters obtained by light and radial velocity curves analysis the absolute parameters of the eclipsing pairs's components recalculated. This results indicate that XY Leo is a W-subtype W UMa binary with a shallow contact degree. The low mass component was found to be brighter than that of similar mass main squence stars. This discrepancy seems to confirm the Wang's (1994) explanation of W-type phenomenon.

The new times for mid-primary and secondary eclipses obtained by us were added to previously published data and the differences from the linear light elements were analyzed. Since the $\mathrm{O}-\mathrm{C}$ residuals for both eclipses show a periodic change superimposed on a parabolic variation we represented the $\mathrm{O}-\mathrm{C}$ curve with Eq. (2). Regular periodic change was attributed to a 
third-body orbit and least-squares solution gave the parameters of the light-time orbit. This analysis shows that the eclipsing pair revolves around a third body star with a period of $19.6 \mathrm{yr}$. The resulting orbital parameters are close to that found by Pan \& Cao (1998) but the eccentricity of 0.12 is lower than the value of 0.17 given by them. We also find relatively larger semimajor axis of the orbit. The reason for the difference is that we used a larger data set spanning about three period cycles of the third body motion. In addition, we included a parabolic change of the orbital period to the analysis of $\mathrm{O}-\mathrm{C}$ curve.

We estimated the mass of the third component as $0.98 M_{\odot}$ for an inclination of $90^{\circ}$. Since the light curve analysis shows a small light contribution from third body to the light of the system its mass should be small, then we assumed that its orbit is nearly perpendicular to the plane of sky. Our analysis yields that the masses of the third and fourth components are about 0.57 and $0.41 M_{\odot}$, with an orbital inclination of about $29^{\circ}$. The separation between two double stars is about 9.6 AU which corresponds to 0 '. 15 for the distance of $63 \mathrm{pc}$ given in the Hipparcos/Tycho catalogue. The oscillatory proper motion of the binary should then be observable when a suitably long sequence of astrometric positions will be available. From the quadratic term in Eq. (2), which is statistically significant, we estimate $\mathrm{d} P / \mathrm{d} t=2.7 \times 10^{-8} \mathrm{day} / \mathrm{yr}$ which corresponds to increment in the orbital period of about 0.23 s per century. A continuous period increase may be due to mass transfer from the less massive component to the more massive one or due to an additional component dynamically bounded to the quadrupole system. Similar period changes may also be produced by magnetic activity, but in a cyclic behaviour. Since the magnetic activity indicators for the XY Leo system is not strong (Barden 1987), the period change might not be connected to solar type activity. If the period change is due to mass transfer, one can easily estimate the mass transfer rate from the less massive component to the more massive one as $4 \times 10^{-8} M_{\odot} / \mathrm{yr}$.

Acknowledgements. K.Y. would like to thank very much to Ercüment UYGUR and M. Yekta VARNALI for their invaluable support. This work is supported by Ege University Research Fund (Project No. 99/FEN/016 and 2002/FEN/002). The authors thank to the referee Dr. Jordi for valuable advice and suggestions.

\section{References}

Agerer, F., \& Heubscher, J. 1996, IBVS, No. 4383 Agerer, F., \& Heubscher, J. 1997, IBVS, No. 4472 Agerer, F., \& Heubscher, J. 1998, IBVS, No. 4562
Agerer, F., \& Heubscher, J. 1999, IBVS, No. 4711

Agerer, F., \& Heubscher, J. 2000, IBVS, No. 4912

Al-Naimiy, H. M. K. 1978, Ap\&SS, 53, 181

Ashbrook, J. 1952, AJ, 57, 63

Ashbrook, J. 1953, AJ, 58, 171

Barden, S. C. 1987, ApJ, 317, 333

Burchi, R., \& Zavatti, F. 1975, IBVS, No. 964

Dorman, B., Nelson, L. A., \& Chau, W. Y. 1989, ApJ, 342, 1003

Faulkner, D. R., \& Grossoehme, D. H. 1983, IBVS, No. 2335

Faulkner, D. R. 1986, PASP, 98, 690

Gehlich, U. K., Prolss, J., \& Wehmeyer, R. 1972, A\&A, 18, 477

Hilditch, R. W. 1981, MNRAS, 196, 305

Hinderer, F. 1960, Observateurs, Marseille, 43, 161

Hoffmeister, C. 1934, Astron. Nachr., 253, 193

Hrivnak, B. J. 1985, ApJ, 290, 696

Hrivnak, B. J., Milone, E. F., Hill, G., \& Fisher, W. A. 1984, ApJ, 285, 683

Kaluzny, J., \& Pojmanski, G. 1982, IBVS, No. 2181

Kaluzny, J., \& Pojmanski, G. 1983, Acta Astron., 33, 277

Keskin, V., \& Pohl, E. 1989, IBVS, No. 3355

Kiss, L. L., Kaszas, G., Füresz, G., \& Vinkó, T. 1999, IBVS, No. 4681

Koch, R. H. 1956, AJ, 61, 47

Koch, R. H. 1960, AJ, 65, 374

Koch, R. H., \& Shanus, C. R. 1978, AJ, 83, 1452

Krzesinski, J., Kuczawska, E., \& Kurpinska-Winiarska, M. 1990, IBVS, No. 3458

Kwee, K. K., \& van Woerden, H. 1956, Bull. Astr. Inst. Netherlands, 12,327

Locher, K. 1973, BBSAG Bul, 7

Lucy, L. B. 1967, Zs. Ap., 65, 89

Lucy, L. B. 1976, ApJ, 342, 1003

Lucy, L. B., \& Wilson, R. E. 1979, ApJ, 231, 502

Markova, L. T. 1994, IBVS, No. 4128

Mullis, C. R., \& Faulkner, D. R. 1991, IBVS, No. 3593

Ogloza, W. 1997, IBVS, No. 4534

Ogloza, W., Drozdz, M., \& Zola, S. 2000, IBVS, No. 4877

Pan, L., \& Cao, M. 1998, Ap\&SS, 259, 285

Pohl, E., \& Kızılırmak, A. 1977, IBVS, No. 1358

Pohl, E., \& Gülmen, Ŏ. 1981, IBVS, No. 1924

Pohl, E., Tunca, Z., Gülmen, O., \& Evren, S. 1985, IBVS, No. 2793

Pohl, E., Akan, M. C., İbanoğlu, C., Sezer, C., \& Güdür, N. 1987, IBVS, No. 3078

Popper, D. M. 1980, ARA\&A, 18, 115

Rucinski, S. M. 1969, Acta Astron., 19, 245

Struve, O., \& Zebergs, V. 1959, ApJ, 130, 137

Wang, J. M. 1994, ApJ, 434, 277

Wilson, R. E., \& Devinney, E. J. 1971, ApJ, 166, 605

Wilson, R. E. 1994, PASP, 106, 921

Wunder, E., Wieck, M., Kılınc, B., et al. 1992, IBVS, No. 3760

Yakut, K., \& İbanoğlu, C. 2001, IBVS, No. 5042

Zessewitch, V. P. 1948, IAU Circ., No. 44, 16 